\title{
On-board Automated Eddy Flux Measurement System over Open Ocean
}

\author{
Satoshi Takahashi ${ }^{1}$, Fumiyoshi Kondo ${ }^{1}$, Osamu Tsukamoto ${ }^{\dagger 2}$, \\ Yoshiki Ito $^{3}$, Seigo Hirayama ${ }^{3}$ and Hiroshi Ishida ${ }^{4}$ \\ ${ }^{1}$ Graduate School of Natural Science and Technology, Okayama University, Okayama, Japan \\ ${ }^{2}$ Department of Earth Sciences, Okayama University, Okayama, Japan \\ ${ }^{3}$ Kaijo Sonic Corp., Tokyo, Japan \\ ${ }^{4}$ Faculty of Maritime Sciences, Kobe University, Kobe, Japan
}

\begin{abstract}
On-board direct eddy flux measurement is essential in the quantitative air-sea flux evaluation as sea truth flux. Present authors have developed a new real-time automated eddy flux system and it is operating as a routine measurement on R/V MIRAI, JAMSTEC. As compared to our previous system, ship motion correction scheme is simplified and real-time eddy flux data processing system is introduced as the automated system. As a continuously operating real time eddy flux ship in the world, this is a unique system.

The quality control of the flux datasets is essential as the data is collected continuously including during unfavorable conditions. Preliminary quality control was applied and the results show the reasonable values as compared to the bulk parameters. Based on the detailed quality control, the bulk transfer coefficients can be parameterized in various conditions.
\end{abstract}

\section{Introduction}

In order to understand the air-sea interaction in the climate system, direct eddy flux measurement is necessary as well as bulk aerodynamic algorithm. However, it is rarely experienced due to many difficulties as described in the next section. The present authors have experienced on-board eddy flux measurement including ship motion correction. Based on their experiences, an automated eddy flux measurement system was developed and can be used without unexperienced scientists. This system includes ship motion correction for the eddy covariance method and real-time, in-situ fluxes are monitored on-board.

This can be applied to many ships cruising global oceans and should be helpful to realize sea surface energy fluxes.
Corresponding author: Department of Earth Sciences, Okayama University, Okayama 700-8530, Japan. E-mail: tsuka@cc.okayma-u.ac.jp. (02005, the Meteorological Society of Japan.

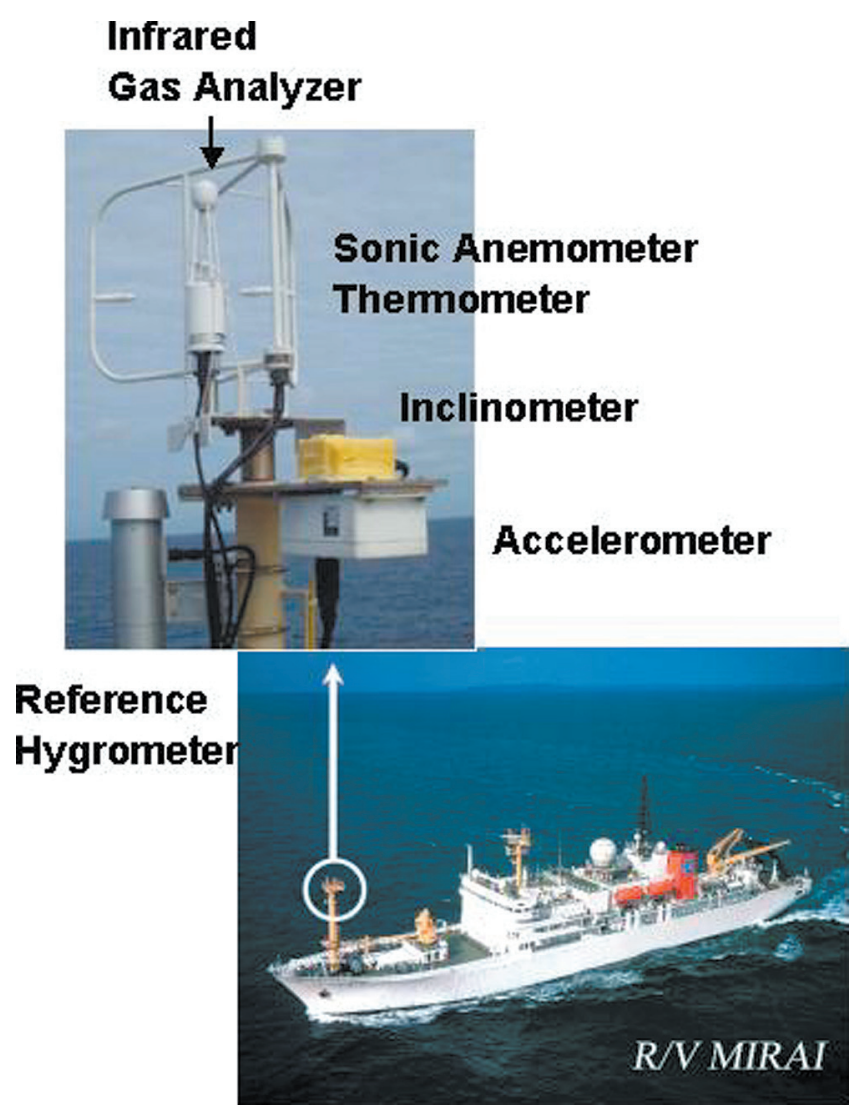

Fig. 1. Eddy flux sensors (upper) on the top of the foremast of R/V MIRAI (lower).

\section{On-board direct eddy covariance system}

Direct eddy covariance system requires fast response turbulence sensors which include a sonic anemometerthermometer and an infrared $\mathrm{H}_{2} \mathrm{O} / \mathrm{CO}_{2}$ analyzer. This kind of eddy covariance system has been applied over land surfaces such as over plant canopies and bare soils including $\mathrm{CO}_{2}$ eddy flux measurements.

However, application of the eddy covariance system over the ocean includes a lot of difficulties and very few measurements are reported as compared to land surface measurements. One of (and the most of) the difficulties 
comes from the moving platform, which is not fixed as over land because the anemometer moves with the platform as the ship moves. The ship motion correction for the wind velocity is a very difficult issue. It was originally developed by Mitsuta and Fujitani (1974). Since then Fujitani (1985), Tsukamoto et al. (1990), Tsukamoto and Ishida (1995) has developed and improved the correction scheme. Around the world, research groups (e.g., Edson et al. 1998; Pedreros et al. 2003) have developed on-board eddy flux system.

\subsection{Revised ship motion correction scheme}

According to Fujitani (1985), the true wind velocity $\left(\mathrm{V}_{\text {true }}\right)$ can be expressed as follows.

$$
V_{\text {true }}=T V_{\text {obs }}+\Omega \times T R+V_{S} .
$$

Here, $V_{\text {obs }}$ is the observed wind velocity relative to the ship coordinate, $\mathrm{T}$ the coordinate transformation matrix, $\Omega$ the angular velocity vector of the ship around the reference coordinate, $\mathrm{R}$ the position vector of the anemometer with respect to the ship coordinate, $V_{\mathrm{S}}$ the translation velocity of the ship with respect to the reference. $V_{\mathrm{S}}$ is expressed as follows using the position vector of the motion sensor, $r$ and the velocity vector of the ship, $\mathrm{V}_{\mathrm{s} 0}$.

$$
V_{S}=V_{S 0}-\Omega \times T r
$$

Then, the final expression is simply written as follows.

$$
V_{\text {true }}=T V_{\text {obs }}+\Omega \times T(R-r)+V_{s 0} .
$$

In the present study, we have developed a new correction scheme. When the ship motion sensor is mounted with the wind vector sensor (i.e., $R=r$ ), the second term of the rhs always fixed to zero. Otherwise, we should know the position vector of the anemometer (R) and the motion sensor (r) from the center of the gravity, which is not easy to fix. In the present study, ship motion sensors were mounted just behind the anemometer as a 'piggyback' system and $\mathrm{R}=\mathrm{r}$ is realized. Then the more simple correction scheme is given as follows.

$$
V_{\text {true }}=T V_{\text {obs }}+V_{s 0}
$$

Here, pitch and roll angles were obtained by a inclinometers and lead to the transformation matrix, $T$. $\mathrm{V}_{\text {obs }}$ is directly measured by the three dimensional sonic anemometer- thermometer and $\mathrm{V}_{\mathrm{s} o}$ is obtained by the time integration of the three axis accelerometers.

\section{Automated eddy flux system on board R/V MIRAI}

The present eddy flux system was introduced on R/V MIRAI, JAMSTEC (Japan Agency for Marine-Earth

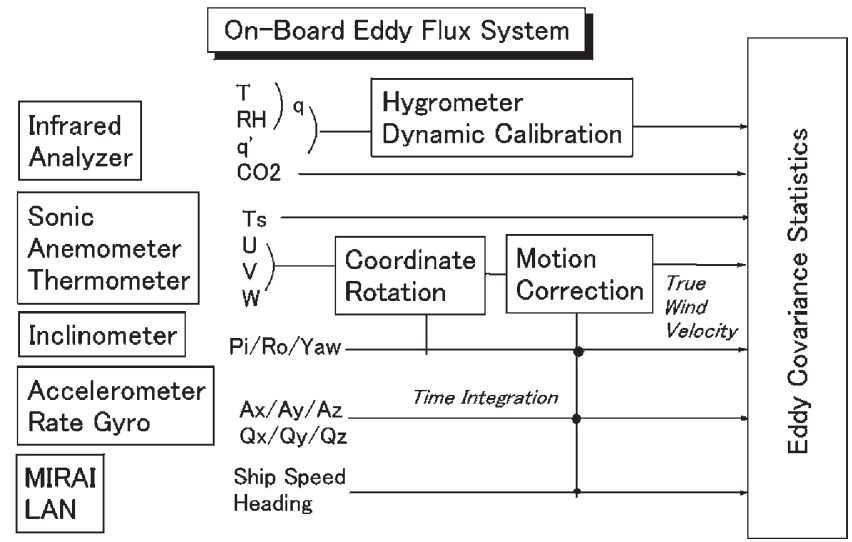

Fig. 2. Block diagram of the real-time data processing system

Science and Technology). On the top of the foremast of $\mathrm{R} / \mathrm{V}$ MIRAI, the present eddy flux system was installed as shown in Fig.1. The MIRAI eddy flux system consists of a 3D sonic anemometer-thermometer (Kaijo DA-600) and an infrared $\mathrm{CO}_{2} / \mathrm{H}_{2} \mathrm{O}$ analyzer (LICOR LI-7500). These turbulence sensors were installed at the top of the foremast to optimally reduce the ship body dynamical and thermal effects. Ship motions were also measured simultaneously by a inclinometer (Applied Geomechanics, MD-900-T) and three axis accelerometers (Applied Signals, QA700-020) and rate gyros (Systron Donners, QRS11-0050-100).

The signals from turbulence sensors and motion sensors were send to a PC based data acquisition system including "LabVIEW" (National Instruments). The system samples all of the data at $10 \mathrm{~Hz}$ and above filtering out high frequency noises and low frequency trends, ship motion correction was applied to the wind velocity data.

The ship motion correction in the LabVIEW system is based on the eq. (4) as the three component equations separately. It is organized as an assembly of subprograms in the system. Practically 10 minutes running means are applied to the motion signals and the motion corrections were operated as the 0.5second averaged turbulence data to minimize the noises. Details of the ship motion correction for the 'piggyback' motion sensor system is described in Takahashi et al. (2000).

For the temperature signal, it is well calibrated and sound virtual temperature effects are corrected. While, for the humidity data, it is reported that the calibration factor can be variable. So we have also included real time calibration unit in the LabVIEW data processing system with a reference hygrometer as a low frequency reference. This is called "dynamic calibration" as mentioned in the next section.. After these correction procedures, 10 minutes eddy fluxes and statistics are obtained in real-time and recorded as well as raw turbulence data. Figure 2 shows the block diagram of the data processing. 


\subsection{Dynamic calibration of the hygrometer}

For the accurate eddy covariance measurements, calibration of the turbulence sensor is very critical. For the sonic anemometer-thermometer, it is well calibrated and its reliability is accepted. The calibration of the hygrometer as a turbulence sensor is rather difficult (Trevitt 1985). Laboratory calibration is not realistic during the continuous long term experiment. The alternative calibration is the dynamic in-situ calibration. It is realized as follows.

A slow response well calibrated reference thermometer/hygrometer was installed with a ventilated radiation shield and mounted near the fast response infrared hygrometer. These slow response signals and fast response signals were put into the turbulence data acquisition system as shown in Fig.2. Combining the slow response air temperature and relative humidity signals, slow response specific humidity time series were obtained. As the slow response sensor is well calibrated in the laboratory, the slow response specific humidity can be regarded as the reference in the low frequency region. Then two specific humidity signals were send to spectral analysis to compare the low frequency variances.

$$
R=\left(\frac{\sum_{f} S(f)_{q \_r e f}}{\sum_{f} S(f)_{q \_I R}}\right)^{1 / 2} .
$$

Here $\Sigma \mathrm{S}(\mathrm{f})_{\mathrm{q}_{\_} \text {ref }}$ and $\Sigma \mathrm{S}(\mathrm{f})_{\mathrm{q}_{\mathrm{IR}} \mathrm{IR}}$ represent the integrated variances of the two hygrometer signals in the calibration band $(0.001-0.01 \mathrm{~Hz})$. As a practical issue, the values of $\mathrm{R}$ can be variable by run to run. The variation can be due to the environmental condition including surrounding temperature and/or humidity or the instrument characteristics (e.g., light intensity, detector sensitivity...). The optimum value is determined by a statistical average and applied to the observed humidity fluctuation in the whole frequency domain. The details of the dynamic calibration is given by Tsukamoto et al. (2001).

\section{Preliminary Results}

$\mathrm{R} / \mathrm{V}$ MIRAI cruises the Western Pacific (from Equatorial to Arctic region) and Eastern Indian Ocean every year. The present eddy flux system is routinely operating in her whole cruises. When these kind of continuous measurements are integrated in these areas, reliable eddy flux database can be established.

\subsection{Quality Control}

When a large amount of continuous measurement data are obtained, 'quality control' of the flux data is important. In some cases, precipitation leads to unfavorable humidity signals, as the glass window of the infrared hygrometer is contaminated by raindrops. In other cases, unfavorable wind direction can increase the ship

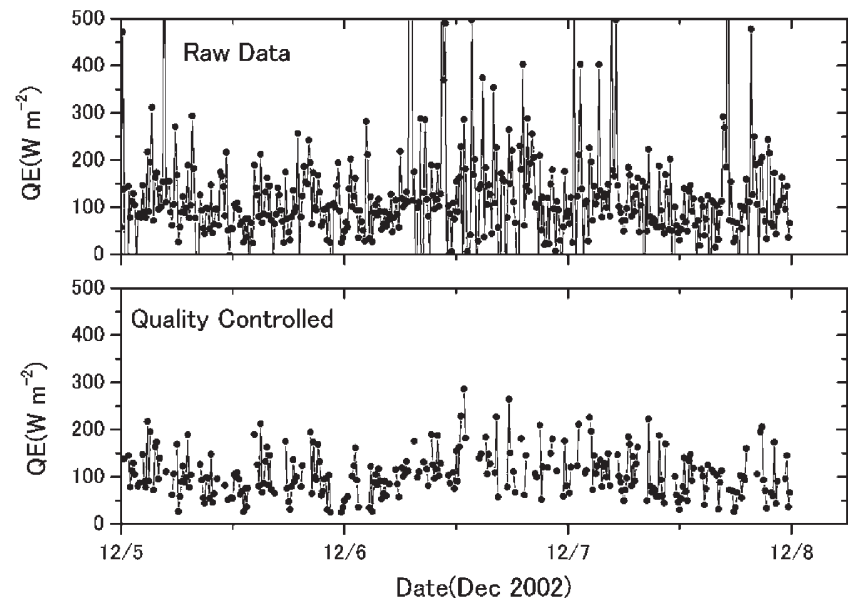

Fig. 3. An example of the quality control for latent heat flux. The eddy fluxes are calculated every 10 minutes.

body effects. Following criterions are applied in the present quality control.

(1) relative wind condition: The best condition for the flux measurement is the ship steaming against the wind as the ship body effect can be minimized. In the present quality control, relative wind speed and direction were checked against the critical values.

(2) ship operation: In the present ship motion correction scheme, the ship should keep the speed and heading during the sampling duration (10 minutes).

(3) instrumental noises: Standard deviations of wind velocity components, temperature and humidity were checked against the critical values to detect the noises due to weather conditions and instrumental malfunctions.

In the present analysis, eddy covariances and statistics were calculated every 10 minutes and sent to the quality control. Figure 3 shows an example of the quality control for the latent heat flux. In the original 10 minutes data, a lot of spikes or noises are observed. While the quality controlled data shows the rather smooth trend as compared to the raw data. The remaining scatter in the latent heat flux can be due to the low frequency (large eddy) contribution.

Based on a preliminary quality control, surface fluxes are evaluated and it is plotted as a function of bulk parameter, $\mathrm{U}\left(\mathrm{q}_{0}-\mathrm{q}\right)$ as shown in Fig.4. Here $\mathrm{q}_{0}$ is the saturation specific humidity at SST including the salinity correction and $\mathrm{q}$ is the specific humidity at the top of the foremast. The latent heat flux is expressed as follows in the eddy-covariance scheme and bulk formula.

$$
Q E=\rho \lambda \overline{w^{\prime} q^{\prime}}=\rho \lambda C_{E} U\left(q_{0}-q\right)
$$

Here, $\rho$ is the air density and $\lambda$ is the latent heat of 


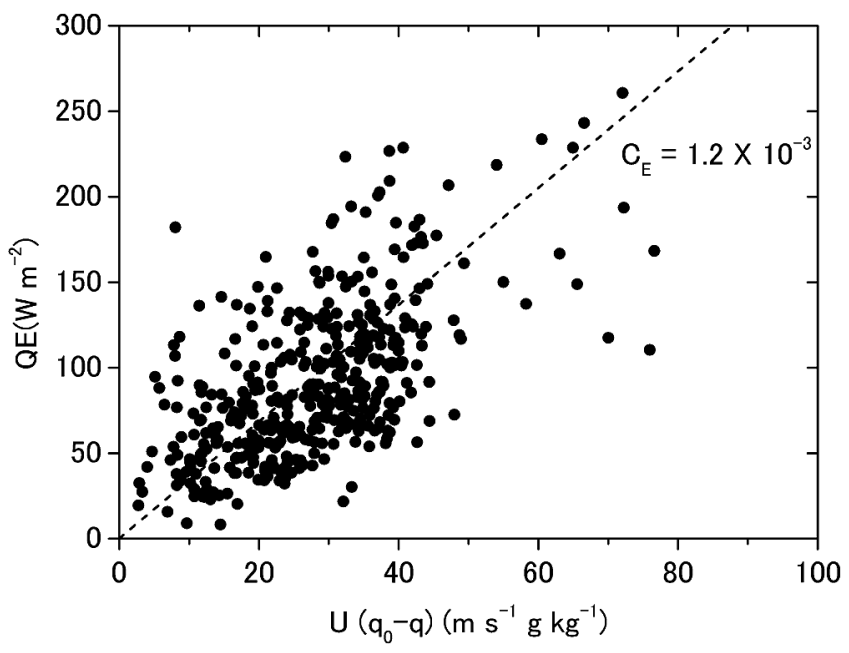

Fig. 4. Latent heat eddy flux as a function of bulk parameter, $\mathrm{U}\left(\mathrm{q}_{0}-\mathrm{q}\right)$. The dotted line corresponds to the bulk transfer coefficient of $\mathrm{C}_{\mathrm{E}}=1.2 \times 10^{-3}$.

evaporation. As a very rough estimate, the bulk transfer coefficient of $\mathrm{C}_{\mathrm{E}}=1.2 \times 10^{-3}$ corresponds to the eddy covariance latent heat flux. According to the previous studies of $\mathrm{C}_{\mathrm{E}}$ based on the eddy-covariance measurements (e.g., Pedreros et al. 2003), the value ranged between $1.1-1.2 \times 10^{-3}$ and the present preliminary result is almost consistent with them. The transfer coefficient can be variable with the wind speed or atmospheric stability, however, the dependency was not observed in the present data.

\section{Concluding Remarks}

In the present study, we have developed a new onboard eddy flux system. It is able to monitor the eddy fluxes in real-time on-board. Then the sea surface fluxes can be monitored as well as normal meteorological parameters.

The importance of on-board eddy-covariance measurement should be pointed out as it is the unique method without any assumptions and experimental factors. Over land surface experiments, this is considered as the global standard including $\mathrm{CO}_{2}$ flux. The application over the sea surface is strongly recommended and more efforts should be given as a global project.

At present, it is not easy to use the on-board eddycovariance method for most of the air-sea flux scientists. The possible way to be a more familiar technique requires a simple automated system that anybody can use without technical skills of eddy flux measurements including ship motion correction. The present study can be one of the systems.

It is still a developing system at present. The following issues remain for future study.

(1) Application to a variety of ship operations with favorable wind conditions

(2) Simple quality control to evaluate the reasonable fluxes

(3) Reasonable parameterization of the bulk transfer coefficients in various seasons and areas

(4) Simple and inexpensive eddy flux sensors for installation

The present system have now included the sea surface $\mathrm{CO}_{2}$ flux measurement system and it is considered to be a important basis for the global warming (Tsukamoto et al. 2004).

\section{Acknowledgements}

The present authors expresses their hearty thanks to all crews of R/V MIRAI lead by Captains Akamine and Hashimoto. They are also indebted to the staff of JAMSTEC and Global Ocean Development Inc. (GODI). Special thanks are given to Dr. Yoneyama in JAMSTEC for his difficult arrangements for on-board measurements. A part of the present study was supported by the Grant-in-Aid for Scientific Research (B) No.13554014 from Japan Society for Promotion of Science (JSPS) and the Mitsubishi Foundation.

\section{References}

Edson, J.B., A.A. Hinton, K.E. Prada, J.E. Hare and C.W. Fairall, 1998: Direct covariance flux estimates from mobile platform at sea, J. Atmos. Oceanic Tech., 15, 547-562.

Fujitani, T, 1985: Method of turbulent flux measurements on a ship by using a stable platform system, Papers in Meteorology and Geophysics, 36, 157-170.

Mitsuta, Y., and T. Fujitani, 1974: Direct measurement of turbulent fluxes on a cruising ship, Boundary-Layer Meteor., 6, 203-217.

Pedreros, R., G. Dardier, H. Dupuis, H.C. Graber, W.M. Drennan, A. Weill, C. Guerin, and P. Nacass, 2003: Momentum and heat fluxes via the eddy correlation method on the R/V LAtlante and an ASIS buoy., J. Geophys. Res., 108, NO.C11, 3339, doi:10.1029/2002JC001449.

Trevitt, A.C.F., 1985: An infrared hygrometer with on-line temperature compensation, Boundary-Layer Meteor., 34, 157169.

Takahashi, T., M. Nabekura, O. Tsukamoto, T. Iwata, T. Takemi and H. Ishida, 2000: Sea surface heat flux evaluation by on-board technique over tropical western Pacific, UMI TO SORA, 76, 79-84.

Tsukamoto, O., E. Ohtaki, H. Ishida, M. Horiguchi and Y. Mitsuta, 1990: On-board direct measurements of turbulent fluxes over the open sea, J. Meteor. Soc. Japan, 68, 203-211.

Tsukamoto, O., and H. Ishida, 1995: Turbulent flux measurements and energy budget analysis over the equatorial Pacific during TOGA-COARE IOP, J. Meteor. Soc. Japan, 73, 557-568.

Tsukamoto O., N, Monji and Y. Ito, 2001: Turbulent flux measurements of momentum, heat and water vapor, Surface flux measurements, Meteor. Res. Notes. No.199, Japan Meteorological Agency (in Japanese).

Tsukamoto, O., S. Takahashi, T. Kono, E. Yamashita, A. Murata and $\mathrm{H}$. Ishida, 2004: Open-path $\mathrm{CO}_{2}$ eddy flux measurement over open ocean, submitted to Boundary-Layer Meteorology.

(Manuscript received 8 December 2004, accepted 4 February 2005) 